\title{
Tissue Compatibility of Two Biodegradable Tubular Scaffolds Implanted Adjacent to Skin or Buccal Mucosa in Mice
}

\author{
D.J. AFRAMIAN, D.M.D., M.Sc., ${ }^{1}$ R.S. REDMAN, D.D.S., Ph.D., 5 \\ S. YAMANO, D.D.S., Ph.D., ${ }^{1}$ J. NIKOLOVSKI, M.S., ${ }^{6}$ E. CUKIERMAN, Ph.D., \\ K.M. YAMADA, M.D., Ph.D., ${ }^{2}$ M.F. KRIETE, D.V.M., ${ }^{3}$ W.D. SWAIM, Ph.D., ${ }^{4}$ \\ D.J. MOONEY, Ph.D., ${ }^{6}$ and B.J. BAUM, D.M.D., Ph.D. ${ }^{1}$
}

\begin{abstract}
Radiation therapy for cancer in the head and neck region leads to a marked loss of salivary gland parenchyma, resulting in a severe reduction of salivary secretions. Currently, there is no satisfactory treatment for these patients. To address this problem, we are using both tissue engineering and gene transfer principles to develop an orally implantable, artificial fluidsecreting device. In the present study, we examined the tissue compatibility of two biodegradable substrata potentially useful in fabricating such a device. We implanted in Balb/c mice tubular scaffolds of poly-L-lactic acid (PLLA), poly-glycolic acid coated with PLLA (PGA/PLLA), or nothing (sham-operated controls) either beneath the skin on the back, a site widely used in earlier toxicity and biocompatibility studies, or adjacent to the buccal mucosa, a site quite different functionally and immunologically. At 1, 3, 7, 14, and 28 days postimplantation, implant sites were examined histologically, and systemic responses were assessed by conventional clinical chemistry and hematology analyses. Inflammatory responses in the connective tissue were similar regardless of site or type of polymer implant used. However, inflammatory reactions were shorter and without epithelioid and giant cells in sham-operated controls. Also, biodegradation proceeded more slowly with the PLLA tubules than with the PGA/PLLA tubules. No significant changes in clinical chemistry and hematology were seen due to the implantation of tubular scaffolds. These results indicate that the tissue responses to PLLA and PGA/PLLA scaffolds are generally similar in areas subjacent to skin in the back and oral cavity. However, these studies also identified several potentially significant concerns that must be addressed prior to initiating any clinical applications of this device.
\end{abstract}

The ${ }^{1}$ Gene Therapy and Therapeutics Branch, ${ }^{2}$ Craniofacial Developmental Biology and Regeneration Branch, ${ }^{3}$ Animal Care Unit, and ${ }^{4}$ Cellular Imaging Core Facility, National Institute of Dental and Craniofacial Research, National Institutes of Health, Bethesda, Maryland.

${ }^{5}$ Oral Pathology Research Laboratory, Department of Veterans Affairs Medical Center, Washington, D.C.

${ }^{6}$ Department of Biomedical Engineering, University of Michigan, Ann Arbor, Michigan. 


\section{INTRODUCTION}

$\mathbf{E}$ XTREME SALIVARY HYPOFUNCTION is a common side effect caused by radiation therapy for cancer in the head and neck region. If the therapeutic radiation treatment exceeds $\sim 50 \mathrm{~Gy}$, generally irreversible damage occurs to the salivary glands. ${ }^{1}$ Indeed, recent studies suggest that a mean dose of 26 Gy should be the planning goal if substantial sparing of salivary glands in the radiation field is desired. ${ }^{2}$ Salivary gland damage leads to persistent hypofunction with marked reductions in salivary output. ${ }^{3}$ Unfortunately, at present, there is no satisfactory treatment for this condition. Affected patients suffer from rampant dental caries, frequent mucosal infections, and dysphagia, as well as considerable pain and discomfort, all of which significantly decrease their quality of life.

We recently initiated an effort to develop an artificial salivary gland device utilizing tissue engineering combined with gene transfer methodology. ${ }^{4-6}$ This device as initially designed consists of a blind end tube fabricated from a biodegradable polymer, coated with a suitable extracellular matrix protein, and lined on its lumen by a monolayer of epithelial cells capable of unidirectional fluid secretion. ${ }^{6}$ An important consideration toward the in vivo application of such a device is tissue compatibility. Many previous studies have employed tissue engineering approaches to replace a variety of tubular tissue structures, including intestine, ${ }^{7}$ blood vessels,${ }^{8}$ ureters, ${ }^{9}$ and trachea. ${ }^{10}$ However, there have been no attempts to study such structures in the orofacial region.

Poly-L-lactic acid (PLLA) and poly-glycolic acid (PGA) have been used in medical devices for over 30 years and are generally considered as biocompatible. ${ }^{1-13}$ This suggested to us that tubular structures made of these materials might be suitable for use in the development of an artificial salivary gland. ${ }^{6}$ In order to test this possibility, we compared the tissue compatibility of tubules made of PLLA, or of PGA coated with PLLA, at two sites. One site, subjacent to the skin on the back, has been widely used in earlier studies by others for examining tissue compatibility of polymer materials. ${ }^{13}$ The other site, subjacent to the buccal mucosa, is the intended functional locale for the artificial salivary gland device. This site differs considerably from those previously examined with these polymers in several important respects. The epithelium of oral mucosa, including the keratinized regions, is much more permeable to a variety of biological agents to which it is frequently exposed (e.g., microbial, food-associated, environmental), and is richer in Langerhans cells, than is that of skin. ${ }^{14,15}$ The oral mucosa is constantly bathed in saliva, while the skin is rarely moistened. Importantly, this bathing fluid can facilitate retrograde access of antigens to, and processing by, minor salivary glands and other epithelial cells. ${ }^{16}$ The intraoral environment also gives rise to a microbial population that is different from, and much richer than, that of skin. ${ }^{17}$

Additionally, the tissue beneath the buccal mucosa differs significantly from the tissue subjacent to the skin on the back because it is a target site for the mucosal immune system. The mucosal immune system is separate from its systemic counterpart, with distinct functions in host defense and differences in major effector mechanisms. ${ }^{18-20}$ For example, at mucosal surfaces the principal antibody present is secretory IgA, while the major immunoglobulin in serum is IgG. Mucosal CD4 ${ }^{+}$(T helper) lymphocytes reflect a bias towards the production of Th2 (e.g., interleukins-4, -5, -6 and -10), versus Th1 (interleukin-2 and interferon- $\gamma$ ) cytokines. ${ }^{21,22}$ Further, mucosal-associated lymphoid cells tend to recirculate within the mucosal system, reflecting a homing mechanism mediated via the differential expression of requisite attachment molecules by postcapillary venules..$^{20,23,24}$

Thus, host responses at sites subjacent to skin and mucosa have the potential to differ in several important respects. Since previous work in which polymers were placed at sites subjacent to the skin on the back might not identify tissue reactions at sites subjacent to oral mucosa, it seemed prudent to examine both of these regions through the present experimental design. For these studies, Balb/c mice were chosen as the in vivo experimental model, because there is extensive information on their genetic and immunologic characteristics, as well as considerable experience with their use in tissue engineering and gene transfer studies. ${ }^{25-27}$ In the present study, we examined host tissue responses using an implanted tubular substratum, constructed of either PLLA alone or of PGA coated on the external surface with PLLA (PGA/PLLA). 


\section{MATERIALS AND METHODS}

\section{Fabrication of PLLA tubes}

Hollow tubes of PLLA (Resomer L206; B.I. Chemicals Inc., Petersburg, VA) were formed by a two-step process. Porous films of the polymers were first fabricated, and thereafter were formed into hollow tubes, using modifications of methods reported previously. ${ }^{12}$ Briefly, to fabricate porous films, the polymer was dissolved in chloroform yielding a $1.56 \%$ solution $(\mathrm{w} / \mathrm{v})$. An $8-\mathrm{mL}$ aliquot of this solution was cast into a 5-cm glass Petri dish covered with a sheet of aluminum-backed tape (Cole Parmer, Chicago, IL). Sieved sodium chloride crystals (150-250 $\mu \mathrm{m}$ in diameter) were dispersed evenly over the solution $(0.375 \mathrm{~g}$ $\mathrm{NaCl} /$ dish), and the chloroform was allowed to evaporate at room temperature. A polymer film with entrapped $\mathrm{NaCl}$ particles resulted. The salt particles were leached out of the film by immersion in one liter of deionized water for $24 \mathrm{~h}$ at room temperature with constant shaking. This procedure yielded a highly porous, thin membrane. Sections were cut from these films and rolled around a 22-gauge needle. The surfaces of the films that were adjacent to the aluminum-backed tape during preparation were always placed adjacent to the needle when fabricating tubules. The overlapping ends of the film were sealed together by briefly exposing one edge to chloroform, and manually pressing the overlapping ends together. This process produced tubes $\sim 3 \mathrm{~mm}$ long, with an inner diameter of $\sim 0.5 \mathrm{~mm}$ (Fig. 1). Tubes were placed under vacuum to remove residual solvent and sterilized by $\gamma$-irradiation.

\section{Fabrication of PGA tubes spray-coated with PLLA}

PGA fibers (Albany International, Mansfield, MA; approximately $12 \mu \mathrm{m}$ in diameter) were assembled into 2-mm-thick nonwoven arrays. These exhibited a bulk density of $50 \mathrm{mg} / \mathrm{mL}$, a porosity of $\sim 97 \%$, a crystallinity of $57 \%$, and an inherent viscosity of $1.23 \mathrm{dL} / \mathrm{g}$ following processing. The residual monomer content was $<0.3 \%$. Sections of the PGA nonwoven mesh were sealed together around a 22-gauge needle, as above, by briefly spraying one end with a 5\% solution (w/v) of PLLA (B.I. Chemicals) in chloroform. PGA arrays were then coated with PLLA $\left(\mathrm{M}_{\mathrm{w}}\right.$ 102,000) to stabilize the arrays and to maintain their shape following implantation. ${ }^{28}$ The PLLA was only applied to the external side of the PGA, and the thickness

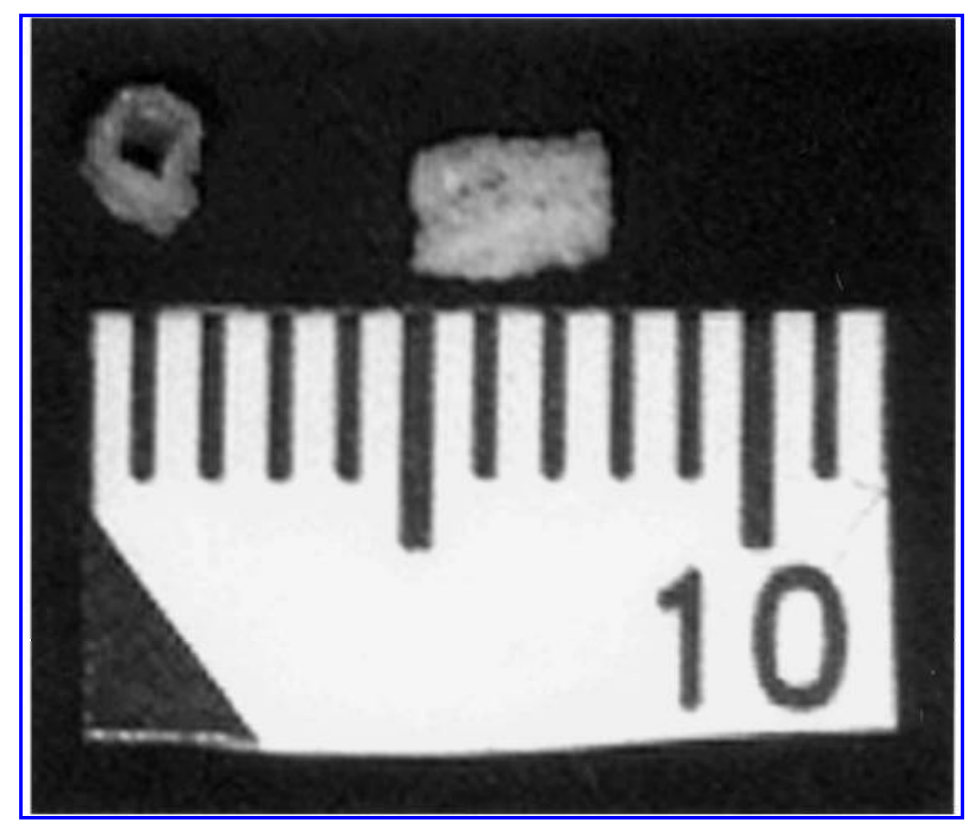

FIG. 1. Photograph of a tubular device fabricated from poly-L-lactic acid (top left, cross section; top right, longitudinal view). 
of PLLA coating was 20-50 $\mu \mathrm{m}$. While still wrapped about the needle, the mesh was held at a distance of $1.5 \mathrm{ft}$ and sprayed uniformly with the PLLA solution for $10 \mathrm{sec}$. The resulting tubes of PGA/PLLA were $\sim 3 \mathrm{~mm}$ long, with an inner diameter of $\sim 0.5 \mathrm{~mm}$. These tubes were placed under vacuum to remove residual solvent and sterilized by $\gamma$-irradiation.

\section{Animals}

One hundred and twenty-four female Balb/c mice ( 8 weeks old) were included in this study. All animals were treated according to procedures approved by the National Institute of Dental and Craniofacial Research Animal Care and Use Committee, and were monitored for any signs of distress. Mice were randomly assigned to seven groups. A zero-time untreated control group $(n=4)$ was used to generate normal clinical chemistry and hematology data for this cohort. The remaining six groups consisted of PLLA implant, PGA/PLLA implant, and sham-operated mice, with surgical sites at either the back or cheek areas. Five time points were assessed (1, 3, 7, 14, and 28 days), and there were four animals/time point/group. Thus, for each experimental and sham-operated group, 20 mice were studied (total =120). Animals were allowed to acclimate for 1 week in our facility before any experimental manipulations were performed. Anesthesia was induced with an intramuscular injection of a mixture of ketamine chloride $(60 \mathrm{mg} / \mathrm{kg})$ and xylazine (5 $\mathrm{mg} / \mathrm{kg}$ ) as previously described. ${ }^{29}$

\section{Implantation procedure}

The back or right cheek area was shaved and disinfected with iodine solution. Thereafter, a small skin incision was made ( $\sim 5 \mathrm{~mm}$ long), and a pocket was created by blunt dissection. Tubes then were implanted via these skin incisions either subcutaneously in the mid-portion of the back or subjacent to the buccal mucosa, that is, the oral mucosal lining of the cheek. Extreme care was taken not to perforate or otherwise damage the buccal mucosa. The incision was closed using 6-0 coated Vicryl absorbable sutures (Ethicon Inc., Somerville, NJ; Fig. 2). It is important to recognize that implantation of tubules beneath the cheek and adjacent to the buccal mucosa in the manner described here does not reflect the intended manner of delivery of an artificial gland device clinically. Ideal placement would be performed intraorally via an incision in the buccal mucosa. For the present study, this was not done because of (i) the small size of the opening

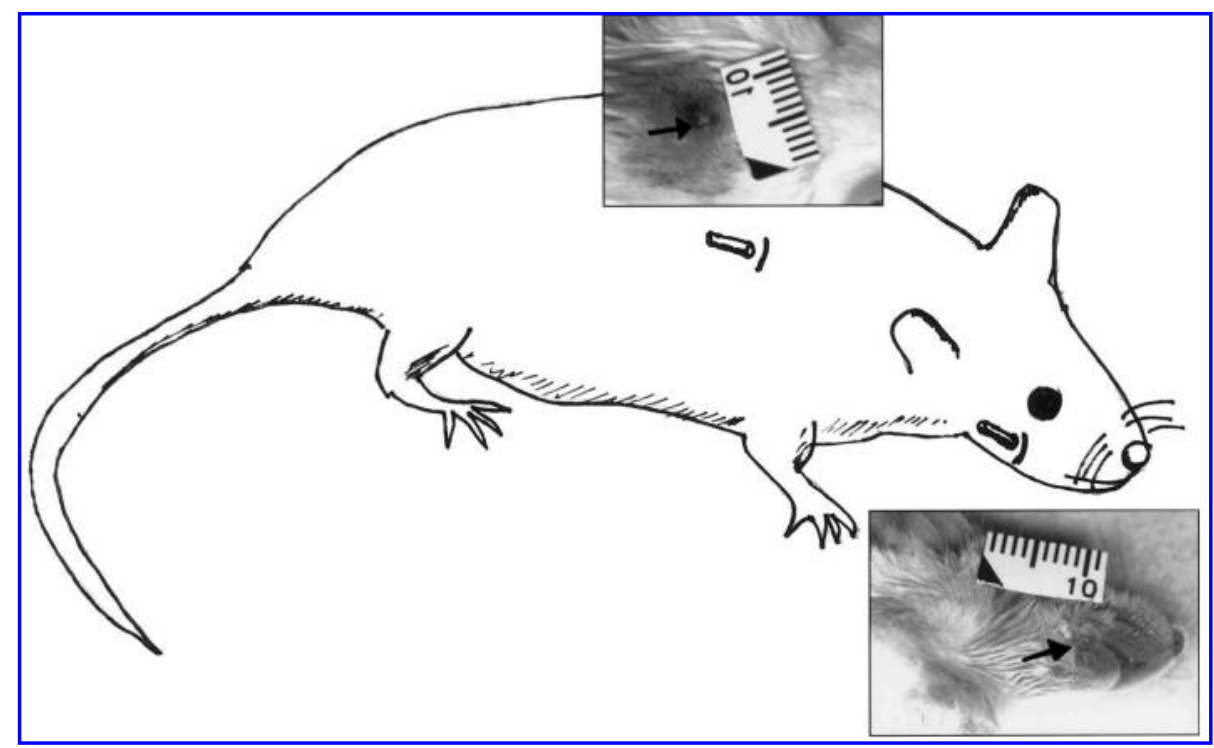

FIG. 2. Illustration depicting the approximate site of tubular device implantation in the back or in the right cheek of a recipient mouse. Photographic inserts show actual implantations at the two tissue target sites. 
between the mouse maxilla and mandible, making such an operative procedure difficult and (ii) our concern that it would be difficult to prevent infection of a significant intraoral wound site in these animals.

At the conclusion of each time point, blood was obtained from the animals by retro-orbital bleeding. Animals then were euthanized by carbon dioxide inhalation. In order to have adequate volumes for all analyses, blood or serum samples from all animals in each separate group, for each separate time point, were pooled. Thus, all clinical chemistry and hematology data reported are average values for the indicated time point within a group. A complete blood count with differential and conventional serum chemistry analyses were performed. In addition, as noted earlier, we utilized another group of age-matched animals, not subjected to any experimental maneuver except obtaining blood, as a control group for hematological and chemistry analyses. Although only representative clinical chemistry results are presented, the following analyses were performed: uric acid, calcium, phosphorous, sodium, potassium, chloride, bicarbonate, magnesium, alkaline phosphatase, total bilirubin, alanine aminotransferase (ALT), aspartate aminotransferase (AST), total protein, albumin, cholesterol, glucose, lactate dehydrogenase, creatinine, urea nitrogen, creatine kinase, triglycerides, amylase, lipase, and gamma-glutamyltransferase.

\section{Histologic examination}

A block of tissue surrounding the implant or sham-operated site, including skin, underlying muscle, and, in the cheek sites, buccal mucosa, was excised from the euthanized animals with a scalpel and scissors. The tissues were fixed immediately in Z-FIX (Anatech, Battle Creek, MI). After paraffin embedding, serial sections $\sim 6 \mu \mathrm{m}$ thick were cut and stained with either hematoxylin and eosin or Masson's trichome connective tissue stain. ${ }^{30}$ Representative photomicrographs were taken using $35 \mathrm{~mm}$ Kodachrome 25 experimental slide film (Eastman Kodak Company, Rochester, NY).

\section{RESULTS}

All but three animals recovered readily from the implantation procedure. Of the three animals that died postoperatively, two had implantations through the cheek adjacent to the buccal mucosa and one received a tubule placed in the back. Two of the tubules implanted in these animals were constructed of PGA/PLLA, and the remaining one was made of PLLA.

\section{Clinical chemistry and hematology}

Blood samples from mice at each time point were pooled together according to each experimental group to provide sufficient sample volume for analyses. Normal values for this study, however, were obtained from individual mice of this cohort that were untreated except for the collection of blood (Fig. 3). For most analyses, there were no major differences seen between the sham-operated and implant groups for any of the measures. Results of four representative analyses are summarized in Figure 3. Neutrophil levels were elevated initially postimplantation of tubules subjacent to the buccal mucosa, above those seen in the shamoperated mice, but returned to similar values by 28 days (Fig. 3A). Lactate dehydrogenase, a cellular enzyme whose serum levels reflect general tissue damage, was high initially in all experimentally manipulated groups and thereafter ( $\sim 14$ days) decreased to low levels (Fig. 3B). The normal control values were also high for this enzyme, likely reflecting trauma associated with the blood collection procedure. Creatinine (a general indicator of renal function) and aspartate aminotransferase (AAT; a general indicator of hepatobiliary function) levels were similar in all groups (implanted and sham; Fig. 3C,D). Initial values of AAT for all groups were high, likely reflecting the effects of anesthesia. In general, the results obtained with mice receiving tubular implants, both PLLA and PGA/PLLA, in either the back or cheek adjacent to the buccal mucosa were quite similar to each other.

\section{Histologic analysis}

PLLA and PGA/PLLA tubules elicited generally similar inflammatory responses at both tissue implantation sites. Initially we observed a moderate to heavy collection of mostly neutrophils and scattered 


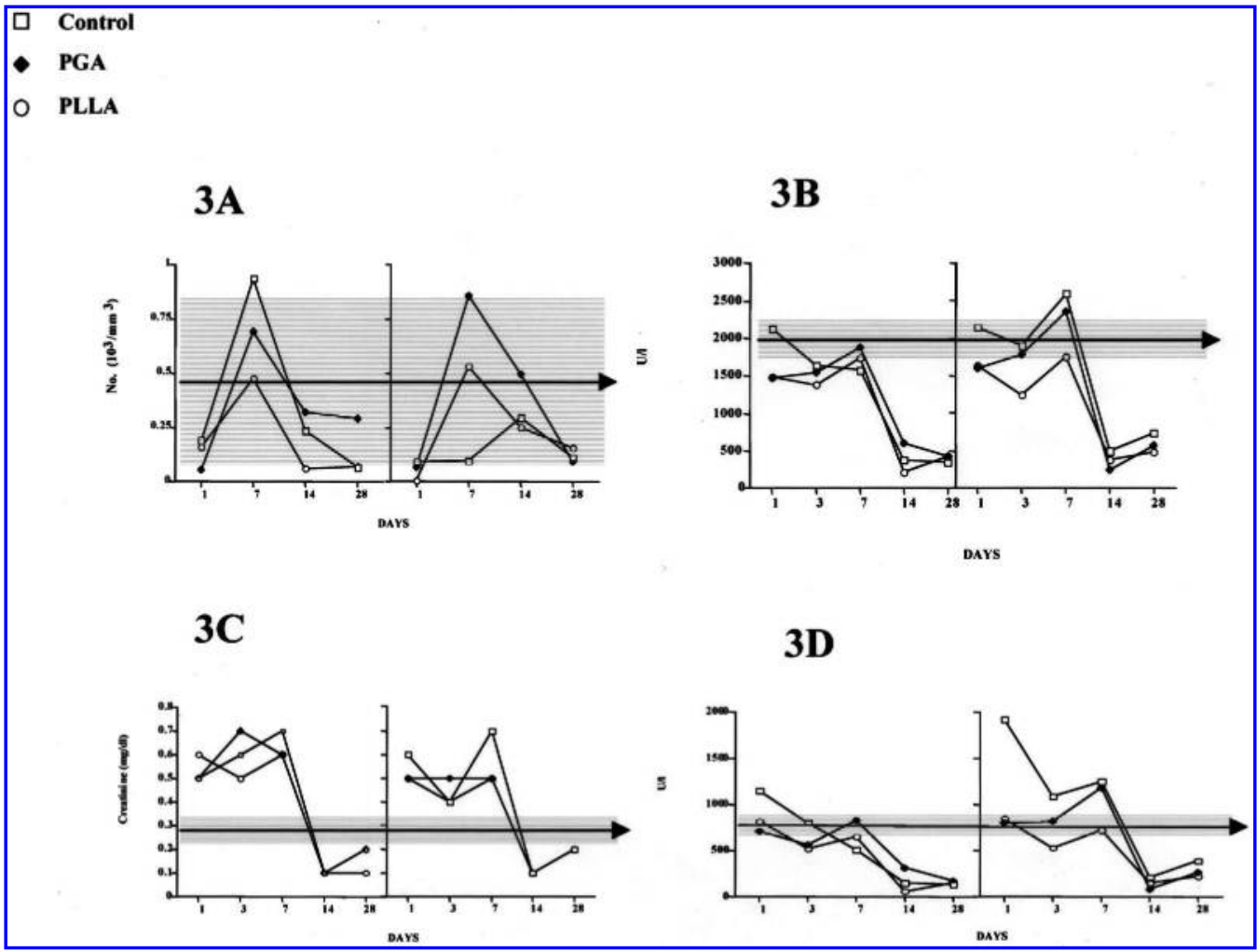

FIG. 3. Representative hematological and clinical chemistry analyses of mice during the 28-day experimental time course. In each panel, the left side represents average results from four mice with sham operations or with tubules implanted in their back, while the right side shows average results from four mice with sham operations or with implants in their cheek. The black arrow in each panel represents the mean values obtained from four individual, nonsurgically treated mice, and the shaded zone represents the standard deviation of those mean values. (A) Neutrophil levels in whole blood. (B) Serum lactate dehydrogenase levels. (C) Serum creatinine levels. (D) Serum aspartate aminotransferase levels.

eosinophils, which gradually was replaced primarily by mononuclear cells (Fig. 4A-C). The chronology of these inflammatory infiltrations was correlated with the elevations in blood neutrophil levels reported above. Beginning at 14 days, the tubules attracted increasing numbers of epithelioid and multinuclear giant cells (Fig. 4D). Over the experimental time course, this resulted in a considerable degradation of the PGA/PLLA tubules (Fig. 4D) at both implant sites. Conversely, the PLLA tubules showed much less degradation over this time, displaying a smooth or only slightly scalloped edge after 28 days (Fig. 4C). The sham-operated sites developed inflammation that was much less intense and much shorter in duration, being essentially resolved after 14 days, with no epitheloid or giant cells being seen.

The skin (both sites) overlying several of the implants developed epithelial hyperplasia, with acanthosis, hyperkeratosis and parakeratosis (Fig. 4E,F). These changes were absent or minimal in later specimens of PGA/PLLA, in which the tubules had been partly digested and the inflammation diminished accordingly. These findings suggest that the skin epithelial changes were a normal, transient response to the stimulus of the underlying inflammation. Oral mucosal epithelium, when observed, appeared unchanged. However, the oral epithelium was missing over areas with an inflammatory response to the implants, and in two places a loose end occurred where the underlying normal tissue approached an inflamed area. This suggested that the epithelium had a weak attachment to the lamina propria in areas of severe inflammation, and thus was stripped off during excision and processing. In addition, we found that three tubules had broken through the skin epithelium after implantation, that is, toward the surgical/tubule implant entrance site. No tubule 

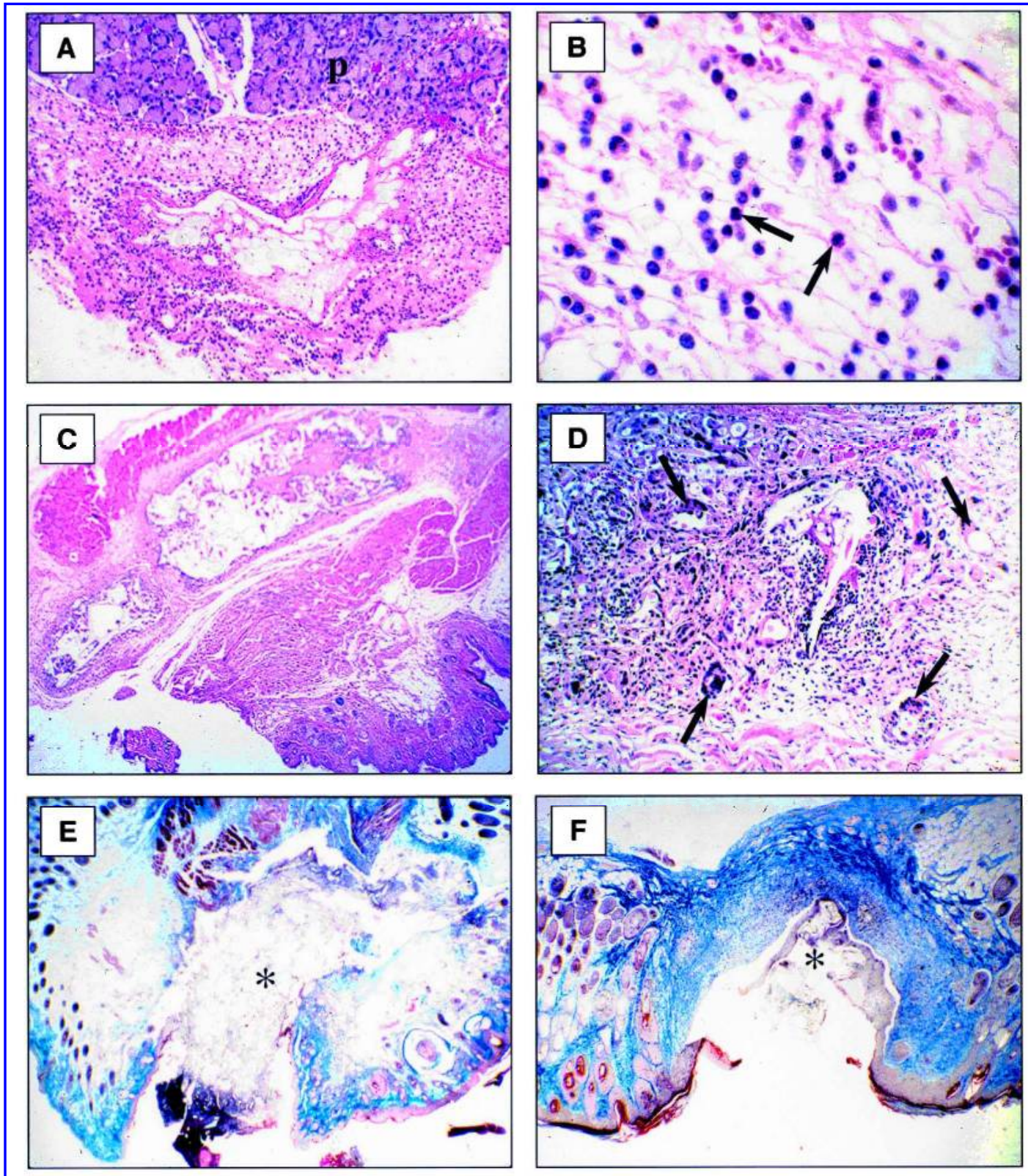

FIG. 4. Representative photomicrographs of inflammatory responses to implanted tubules. (A) PGA/PLLA tubule 1 day after implantation in buccal mucosa, surrounded by inflammatory cells. Oral cavity side is at bottom, with a portion of parotid gland (p) above. (B) Detail of inflammatory response near the implanted tubule in A, showing mostly neutrophils but with scattered eosinophils (arrows). (C) PLLA tubule 28 days after implantation in the back. Despite the inflammation, most of the tubule remains undegraded. Skin is at top. (D) PGA/PLLA tubule 28 days after implantation beneath the buccal mucosa. The scattered remnants of the tubule are surrounded by epithelioid and foreign body giant cells (arrows), as well as by lymphocytes, histiocytes, and neutrophils. (E,F) PGA/PLLA and PLLA tubules (indicated by *) at 3 and 14 days after implantation in the mouth and back, respectively. Both tubules have breached the skin surface, with the epithelium then having migrated part way (E) and completely (F) around the tubules, a process appearing to lead toward exfoliation. The surface epithelium adjacent to the tubule in F is hypertrophic, with acanthosis and hyperkeratosis, but no dysplasia. Stains: hematoxylin and eosin (A-D), trichrome (E,F). Magnifications, $\times 21$ (A,C,E,F), $\times 310(\mathrm{~B}), \times 80(\mathrm{D})$. 
was seen to extrude toward the buccal mucosa. In these specimens, the epithelium had migrated progressively along the external faces of the tubules (Fig. 4E,F), a process that appeared to be leading to exfoliation.

\section{DISCUSSION}

Our long-range objective is to utilize an artificial substratum to deliver organized epithelial cells into the oral mucosa under conditions where the cells will thrive and secrete a saliva-like fluid. The resulting artificial salivary gland could supplement or otherwise enhance salivary secretions for individuals whose salivary glands have been seriously and permanently damaged. ${ }^{31}$ In the present study, we have assessed the biocompatibility, inflammatory responses, and general systemic response characteristics of two candidate matrices for such an artificial gland, PLLA and PGA/PLLA, in a frequently used animal model, Balb/c mice.

We found that the local tissue reaction to both polymers was more intense and prolonged than that which was seen with the sham-operated mice. This elevated inflammatory reaction was observed at both implant sites; i.e., in both the back and buccal mucosa. Systemic reactions were minimal, with no significant differences generally found between animals with sham-operations and tubule implants. The local tissue reactions to PLLA and PGA/PLLA tubes were essentially identical, and the progression from acute to more chronic inflammation was similar to that described in the literature. ${ }^{12,32-35}$ However, because of the animal model tested, we were unable to implant the tubules in a manner directly comparable to that which we anticipate would be done clinically. It will be necessary to perform preclinical animal studies in a model substantially larger than mice, despite the immunological and genetic value of the latter.

We did observe one potentially very important difference between the polymer implants: the much slower rate of biodegradation of PLLA compared with PGA/PLLA at both implant sites in vivo. Such differences in rates of degradation with these and other polymers have been reported in vivo. ${ }^{34,36}$ If the tubular scaffold is essential to maintain substantive structural integrity for the early postplacement function of the proposed artificial salivary gland, for example, to prevent collapse of the orifice or to maintain differentiation of the lining cells, then the slower rate of degradation of PLLA tubules is advantageous. On the other hand, it is conceivable that as the tubule is digested and removed, the salivary cells might become established in a tubule-shaped monolayer on the granulation tissue bed left behind and join with the oral epithelium at the orifice, providing for long-term function. In either case, however, the inflammatory response to both tubules may be long and intense enough to damage or destroy the lining salivary cells, another potentially significant concern.

Regardless of whether or not the salivary cells require permanent support of a tubular scaffold, their satisfactory functioning will depend on the adequacy of their blood supply and an appropriate level of neuronal stimulation. The oral mucosa is well supplied with blood vessels, and angiogenesis likely would be enhanced by the initial inflammatory response. However, in irradiated tissue it may prove necessary to facilitate angiogenesis ${ }^{37}$ adjacent to implanted tubules, because the vasculature may be greatly reduced by endothelial cell death and by subsequent scarring. ${ }^{31}$ Innervation may be more difficult to attain, particularly in the irradiated fibrotic gland in the absence of a neurotrophic stimulus. ${ }^{38}$

A further consideration with irradiated tissues is the timing of clinical implantation of the device. Following irradiation, an oral mucositis often develops. ${ }^{39}$ During this period, the oral mucosa can be erythematous, ulcerated, and extremely painful. Following completion of radiotherapy, mucosal healing promptly commences. Thus, optimal timing for intraoral implantation of an artificial salivary gland likely would be after resolution of the mucositis (4-6 weeks postirradiation).

In addition, it must be emphasized that the tubules implanted here were devoid of cells. All of our previous analyses of cell and matrix protein interactions with PLLA and PGA were performed in tissue culture with allogeneic graft cells in the absence of any host inflammatory response. Obviously, it is critical to examine such interactions under in vivo conditions. ${ }^{40}$ However, if allogeneic cells were employed in the device, it would likely lead to an additional immune response directed toward the cells. This could be obviated by the use of autologous epithelial cells. The ability to grow and maintain primary cultures of several salivary epithelial cell types has been achieved with rat glands. ${ }^{41,42}$ Clearly, an important next step in 
the development of the envisioned device would be to study the growth of primary epithelial cells on polymer substrata in vivo.

Normal wound healing involves migration of the surface epithelium along granulation tissue as it develops, undermining and ejecting the clot. An analogous type of process seems to have occurred in the three specimens in which the tubule had breached the surface toward the surgical incision site on the skin. This phenomenon may pose a significant problem for the envisioned artificial gland. However, similar problems have already been managed in other intraoral operative situations, for example, surgical reduction of periodontal pockets and prevention of exfoliation of dental implants. For example, in the latter circumstance, the tight mechanical and biological interactions occurring between the bone and implant surfaces appear to provide a barrier preventing epithelial migration apically, as long as the epithelium and supporting lamina propria are $\sim 2 \mathrm{~mm}$ thick, and there is little peri-implant inflammation. ${ }^{43}$ In the case of an artificial gland, in order to provide a patent orifice through which fluid can flow, it will be critical to firmly secure the orifice of the device to the buccal mucosa to prevent its ejection. Future studies will need to specifically address this concern by implanting the device in a manner consistent with its intended clinical use.

The biodegradable polyesters PLLA, PGA, and their copolymers have been extensively used in biomedical applications, and their biocompatibility widely studied. ${ }^{44}$ These polymers have been commercially available since 1970 as surgical sutures, as this was their first approved clinical use. ${ }^{11}$ They are also used in dental and orthopedic surgical procedures as implantable biomaterials. ${ }^{45,46}$ More recently, these polymers have been utilized as cell transplantation vehicles for tissue engineering. ${ }^{47}$ PLLA and PGA are typically considered biocompatible when tested by various in vivo or in vitro assays, ${ }^{44,48}$ although the majority of biocompatibility testing to date has used subcutaneous implantation. ${ }^{13,34}$ The biocompatibility of these polymers in the form of solid implants has been questioned in certain orthopedic applications, ${ }^{45}$ which may be related to local acidosis resulting from device degradation. Furthermore, these polymers in microparticular form can be used to carry antigens, and they appear particularly useful for oral mucosal immunization. ${ }^{49-51}$ The breakdown of both PLLA and PGA/PLLA as shown herein adjacent to the oral mucosa, along with the potential for associated damage to the lining graft cells, could provide a source for local mucosal immune challenge that must be addressed in the future.

In conclusion, we believe that the results presented here represent an important step toward understanding the potential complications following placement of an artificial salivary gland subjacent to the oral mucosa. However, future studies in developing this device clearly require that several problems identified by us herein be addressed in a more suitable animal model system.

\section{REFERENCES}

1. Taylor, S.E., and Miller, E.G. Preemptive pharmacologic intervention in radiation-induced salivary dysfunction. Proc. Soc. Exp. Biol. Med. 221, 14, 1999.

2. Eisbruch, A., Ten Haken, R.K., Kim, H.M., et al. Dose, volume, and function relationships in parotid salivary glands following conformal and intensity-modulatedirradiation of head and neck cancer. Int. J. Radiat. Oncol. Biol. Phys. 45, 577, 1999.

3. Valdez, I.H., and Fox, P.C. Diagnosis and management of salivary dysfunction. Crit. Rev. Oral Biol. Med. 4, 271, 1993.

4. Aframian, D.J., Cukierman, E., Nikolovski, J., et al. The growth and morphological behavior of salivary epithelial cells on matrix protein-coated biodegradable substrata. Tissue Eng. 6, 209, 2000.

5. Aframian, D.J., Zheng, C., Goldsmith, C.M., et al. Using HSV thymidine-kinase for safety in an allogeneic salivary graft cell line. Tissue Eng. 7, 405, 2001.

6. Baum, B.J., Wang, S., Cukierman, E., et al. Re-engineering the functions of a terminally-differentiated epithelial cell in vivo. Ann. N.Y. Acad. Sci. 875, 294, 1999.

7. Organ, G.M., Mooney, D.J., Hansen, L.K., et al. Transplantation of enterocytes utilizing polymer-cell constructs to produce a neointestine. Transplant. Proc. 24, 3009, 1992.

8. Weinberg, C.B., and Bell, E. A blood vessel model constructed from collagen and cultured vascular cells. Science 231, 397, 1986.

9. Atala, A., Freeman, M.R., Vacanti, J.P., et al. Implantation in vivo and retrieval of artificial structures consisting of rabbit and human urothelium and human bladder muscle. J. Urol. 150, 608, 1993. 
10. Robey, T.C., Valimaa, T., Murphy, H.S., et al. Use of internal bioabsorbable PLGA "finger-type" stents in a rabbit tracheal reconstruction model. Arch. Otolaryngol. Head Neck Surg. 126, 985, 2000.

11. Frazza, E.J., and Schmitt, E.E. A new absorbable suture. J. Biomed. Mater. Res. 5, 43, 1971.

12. Mooney, D.J., Breuer, C., McNamara, K., et al. Fabricating tubular devices from polymers of lactic and glycolic acid for tissue engineering. Tissue Eng. 2, 107, 1995.

13. Athanasiou, K.A., Niederauer, G.G., and Agrawal, C.M. Sterilization, toxicity, biocompatibility and clinical applications of polylactic acid/polyglycolic acid copolymers. Biomaterials 17, 93, 1996.

14. Squier, C.A. The permeability of the oral mucosa. Crit. Rev. Oral Biol. Med. 12, 13, 1991.

15. Barrett, A.W., Cruchley, A.T., and Williams, D.M. Oral mucosal Langerhans cells. Crit. Rev. Oral Biol. Med. 7, 36, 1996.

16. Nair, P.C.N., and Schroeder, H.E. Retrograde access of antigens to the minor salivary glands in the monkey Macaca fascicularis. Arch. Oral Biol. 28, 145, 1983.

17. Boackle, R.J. The interaction of salivary secretions with the human complement system-a model for the study of host defense systems on inflamed mucosal surfaces. Crit. Rev. Oral Biol. Med. 2, 355, 1991.

18. Ogra, P.L., Mestecky, J., Lamm, M.E., et al., eds. Mucosal Immunology, 2nd ed. San Diego: Academic Press, 1999.

19. Brandtzaeg, P., Farstad, I.N., Johansen, F.-E., et al. The B-cell system of human mucosae and exocrine glands. Immunol. Rev. 171, 45, 1999.

20. Roitt, I., Brostoff, J., and Male, D. Immunology, 6th ed. Edinburgh: Mosby, pp. 31-75, 2001.

21. Xu-Amano, J., Kiyono, H., Jackson, R.J., et al. Helper T cell subsets for immunoglobulin A responses: oral immunization with tetanus toxoid and cholera toxin as adjuvant selectively induces Th2 cells in mucosa associated tissues. J. Exp. Med. 178, 1309, 1993.

22. Husband, A.J., Beagley, K.W., and McGhee, J.R. Mucosal cytokines. In: Ogra, P.L., Mestecky, J., Lamm, M.E., et al., eds. Mucosal Immunology, 2nd ed. San Diego: Academic Press, pp. 541-557, 1999.

23. Steinman, R.M. Dendritic cells. In: Paul, W.E., ed. Fundamental Immunology, 4th ed. Philadelphia: LippincottRaven, pp. 547-573, 1998.

24. Bienenstock, J., McDermott, M.R., and Clancy, R.L. Respiratory tract defenses: role of mucosal lymphoid tissues. In: Ogra, P.L., Mestecky, J., Lamm, M.E., et al., eds. Mucosal Immunology, 2nd ed. San Diego: Academic Press, pp. 283-292, 1999.

25. Pecquet, S., Leo, E., Fritsche, R., et al. Oral tolerance elicited in mice by beta-lactoglobulinentrapped in biodegradable microspheres. Vaccine 18, 1196, 2000.

26. Dalu, A., Blaydes, B.S., Lomax, L.G., et al. A comparison of the inflammatory response to a polydimethylsiloxane implant in male and female Balb/c mice. Biomaterials 21, 1947, 2000.

27. Maheshwari, A., Mahato, R.I., McGregor, J., et al. Soluble biodegradable polymer-based cytokine gene delivery for cancer treatment. Mol. Ther. 2, 121, 2000.

28. Mooney, D.J., Mazzoni, C.L., Breuer, C., et al. Stabilized polyglycolic acid fibre-based tubes for tissue engineering. Biomaterials 17, 115, 1996.

29. Kagami, H., O’Connell, B.C., and Baum, B.J. Evidence for the systemic delivery of a transgene product from salivary glands. Hum. Gene Ther. 7, 2177, 1996.

30. Luna, L.G. Manual of Histologic Staining Methods of the Armed Forces Institute of Pathology, 3rd ed. New York: McGraw-Hill, pp. 33-39, 94-95, 1968.

31. Kashima, H.K., Kirkham, J.R., and Andrews, J.R. Postirradiation sialadenitis. A study of the clinical features, histopathologic changes and serum enzyme variations following irradiation of human salivary glands. Am. J. Roentgenol. Radium Ther. Nucl. Med. 94, 77, 1965.

32. Bos, R.R., Rozema, F.R., Boering, G., et al. Degradation of and tissue reaction to biodegradable poly(L-lactide) for use as internal fixation of fractures: a study in rats. Biomaterials 12, 32, 1991.

33. Vince, D.G., Hunt, J.A., and Williams, D.F. Quantitative assessment of the tissue response to implanted biomaterials. Biomaterials 12, 731, 1991.

34. Holder, W.D., Jr., Gruber, H.E., Moore, A.L., et al. Cellular ingrowth and thickness changes in poly-L-lactide and polyglycolide matrices implanted subcutaneously in the rat. J. Biomed. Mater. Res. 41, 412, 1998.

35. Ronneberger, B., Kao, W.J., Anderson, J.M., et al. In vivo biocompatibility study of ABA triblock copolymers consisting of poly(L-lactic-co-glycolic acid) A blocks attached to central poly(oxyethylene) B blocks. J. Biomed. Mater. Res. 30, 31, 1996.

36. Pitt, C.G., Gratzl, M.M., Kimmel, G.L., et al. Aliphatic polyesters II. The degradation of poly(DL-lactide), poly(epsilon-caprolactone), and their copolymers in vivo. Biomaterials 2, 215, 1981.

37. Paris, F., Fuks, Z., Kang, A., et al. Endothelial apoptosis as the primary lesion initiating intestinal radiation damage in mice. Science 293, 293, 2001. 


\section{BIOCOMPATIBILITY OF TUBULAR SCAFFOLDS}

38. Heuckeroth, R.O., Enomoto, H., Grider, J.R., et al. Gene targeting reveals a critical role for neurturin in the development and maintenance of enteric, sensory, and parasympathetic neurons. Neuron 22, 201, 1999.

39. Peterson, D.E. Research advances in oral mucositis. Curr. Opin. Oncol. 11, $26 \overline{1,1999 .}$

40. Suggs, L.J., Shive, M.S., Garcia, C.A., et al. In vitro cytotoxicity and in vivo biocompatibility of poly(propylene fumarate-co-ethylene glycol) hydrogels. J. Biomed. Mater. Res. 46, 22, 1999.

41. Quissell, D.O., Redman, R.S., Barzen, K.A., et al. Effects of oxygen, insulin, and glucagon concentrations on rat submandibular acini in serum-free primary culture. In Vitro Cell. Dev. Biol. 30A, 833, 1994.

42. Yeh, C.-K., Mertz, P.M., Oliver, C., et al. Cellular characteristics of long-term cultured rat parotid acinar cells. In Vitro Cell. Dev. Biol. 27A, 707, 1991.

43. Salvi, G.E., and Lang, N.P. Changing paradigms in implant dentistry. Crit. Rev. Oral Biol. Med. 12, 262, 2001.

44. Gilding, D.K. Biodegradable polymers. In: Williams DF, ed. Biocompatibility of Clinical Implant Materials. Boca Raton, FL: CRC Press, pp. 209-232, 1981.

45. Athanasiou, K.A., Agrawal, C.M., Barber, F.A., et al. Orthopaedic applications for PLA-PGA biodegradable polymers. Arthroscopy 14, 726, 1998.

46. Wang, H.L., and MacNeil, R.L. Guided tissue regeneration. Absorbable barriers. Dent. Clin. North Am. 42, 505, 1998.

47. Langer, R., and Vacanti, J.P. Tissue engineering. Science 260, 920, 1993.

48. Ignatius, A.A., and Claes, L.E. In vitro biocompatibility of bioresorbable polymers: poly(L,DL-lactide) and poly(Llactide-co-glycolide). Biomaterials 17, 831, 1996.

49. Kofler, N., Ruedl, C., Rieser, C., et al. Oral immunization with poly-(D,L-lactide-co-glycolide) and poly-(L-lactic acid) microspheres containing pneumotropic bacterial antigens. Int. Arch. Allergy Immunol. 113, 424, 1997.

50. Kim, S.Y., Doh, H.J., Ahn, J.S., et al. Induction of mucosal and systemic immune response by oral immunization with $H$. pylori lysates encapsulated in poly(D,L-lactide-co-glycolide) microparticles. Vaccine 17, 607, 1999.

51. Smith, D.J., Trantolo, D.J., King, W.F., et al. Induction of secretory immunity with bioadhesive poly(D,L-lactideco-glycolide) microparticles containing Streptococcus sobrinus glucosyltransferase Oral Microbiol. Immunol. 15, $124,2000$.

Address reprint requests to:

Bruce J. Baum, D.M.D., Ph.D.

GTTB, NIDCR, NIH

Bldg. 10, Rm. 1N113

MSC-1190

Bethesda, MD 20892

E-mail: bbaum@dir.nidcr.nih.gov 


\section{This article has been cited by:}

1. E. L. SCHELLER, P. H. KREBSBACH, D. H. KOHN. 2009. Tissue engineering: state of the art in oral rehabilitation. Journal of Oral Rebabilitation 36:5, 368-389. [CrossRef]

2. Doron J. Aframian , Aaron Palmon . 2008. Current Status of the Development of an Artificial Salivary GlandCurrent Status of the Development of an Artificial Salivary Gland. Tissue Engineering Part B: Reviews 14:2, 187-198. [Abstract] [PDF] [PDF Plus]

3. Ran David, Ela Shai, Doron J. Aframian, Aaron Palmon . 2008. Isolation and Cultivation of Integrin $\alpha 6 \beta 1-E x p r e s s i n g$ Salivary Gland Graft Cells: A Model for Use with an Artificial Salivary GlandIsolation and Cultivation of Integrin $a 6 \beta 1-$ Expressing Salivary Gland Graft Cells: A Model for Use with an Artificial Salivary Gland. Tissue Engineering Part A 14:2, 331-337. [Abstract] [PDF] [PDF Plus]

4. Min-Huey Chen, Yi-Jane Chen, Chih-Chen Liao, Yen-Hui Chan, Chia-Yung Lin, Rung-Shu Chen, Tai-Hong Young. 2008. Formation of salivary acinar cell spheroids in vitro above a polyvinyl alcohol-coated surface. Journal of Biomedical Materials Research Part A 9999A, NA-NA. [CrossRef]

5. Ran David , Ela Shai , Doron J. Aframian, Aaron Palmon. Isolation and Cultivation of Integrin $\alpha 6 \beta 1-E x p r e s s i n g$ Salivary Gland Graft Cells: A Model for Use with an Artificial Salivary GlandIsolation and Cultivation of Integrin a6 $\beta 1$-Expressing Salivary Gland Graft Cells: A Model for Use with an Artificial Salivary Gland. Tissue Engineering, ahead of print. [Abstract] [PDF] [PDF Plus]

6. Doron J. Aframian, Doron Amit, Ran David, Ela Shai , Dan Deutsch, Alexander Honigman , Amos Panet, Aaron Palmon . 2007. Reengineering Salivary Gland Cells to Enhance Protein Secretion for Use in Developing Artificial Salivary Gland DeviceReengineering Salivary Gland Cells to Enhance Protein Secretion for Use in Developing Artificial Salivary Gland Device. Tissue Engineering 13:5, 995-1001. [Abstract] [PDF] [PDF Plus]

7. Simon D. Tran, Takayuki Sugito, Giovanni Dipasquale, Ana P. Cotrim, Bidhan C. Bandyopadhyay, Kathryn Riddle, David Mooney, Marc R. Kok, John A. Chiorini, Bruce J. Baum. 2006. Re-engineering Primary Epithelial Cells from Rhesus Monkey Parotid Glands for Use in Developing an Artificial Salivary Gland. Tissue Engineering, ahead of print060928130622002. [CrossRef]

8. Simon D. Tran , Takayuki Sugito , Giovanni Dipasquale , Ana P. Cotrim , Bidhan C. Bandyopadhyay, Kathryn Riddle, David Mooney, Marc R. Kok, John A. Chiorini , Bruce J. Baum . 2006. Re-engineering Primary Epithelial Cells from Rhesus Monkey Parotid Glands for Use in Developing an Artificial Salivary GlandRe-engineering Primary Epithelial Cells from Rhesus Monkey Parotid Glands for Use in Developing an Artificial Salivary Gland. Tissue Engineering 12:10, 2939-2948. [Abstract] [PDF] [PDF Plus]

9. Bruce J. Baum, Simon D. Tran. 2006. Synergy between genetic and tissue engineering: creating an artificial salivary gland. Periodontology 2000 41:1, 218-223. [CrossRef]

10. A. P. Marques, H. R. Cruz, O. P. Coutinho, R. L. Reis. 2005. Effect of starch-based biomaterials on the in vitro proliferation and viability of osteoblast-like cells. Journal of Materials Science: Materials in Medicine 16:9, 833-842. [CrossRef]

11. Min-Huey Chen, Rung-Shu Chen, Yuan-Hua Hsu, Yi-Jane Chen, Tai-Horng Young . 2005. Proliferation and Phenotypic Preservation of Rat Parotid Acinar CellsProliferation and Phenotypic Preservation of Rat Parotid Acinar Cells. Tissue Engineering 11:3-4, 526-534. [Abstract] [PDF] [PDF Plus]

12. S.D. Tran , J. Wang, B.C. Bandyopadhyay , R.S. Redman , A. Dutra , E. Pak , W.D. Swaim , J.A. Gerstenhaber, J.M. Bryant, C. Zheng, C.M. Goldsmith, M.R. Kok, R.B. Wellner , B.J. 
Baum . 2005. Primary Culture of Polarized Human Salivary Epithelial Cells for Use in Developing an Artificial Salivary GlandPrimary Culture of Polarized Human Salivary Epithelial Cells for Use in Developing an Artificial Salivary Gland. Tissue Engineering 11:1-2, 172-181. [Abstract] [PDF] [PDF Plus]

13. D.J. Aframian , R. David, H. Ben-Bassat, E. Shai , D. Deutsch , B.J. Baum , A. Palmon . 2004. Characterization of Murine Autologous Salivary Gland Graft Cells: A Model for Use with an Artificial Salivary GlandCharacterization of Murine Autologous Salivary Gland Graft Cells: A Model for Use with an Artificial Salivary Gland. Tissue Engineering 10:5-6, 914-920. [Abstract] [PDF] [PDF Plus] 